International Journal of Bifurcation and Chaos, Vol. 10, No. 5 (2000) 1019-1032

(c) World Scientific Publishing Company

\title{
MODELING CHAOTIC DYNAMICS WITH DISCRETE NONLINEAR RATIONAL MODELS
}

\author{
M. V. CORREA ${ }^{\dagger}$ and L. A. AGUIRRE* \\ Laboratoratório de Modelagem, Análise e Controle de Sistemas Não Lineares, \\ * Departamento de Engenharia Eletrônica, Universidade Federal de Minas Gerais, \\ Av. Antônio Carlos, 6627, 31270-901 Belo Horizonte, M.G., Brazil \\ ${ }^{\dagger}$ Instituto Católico de Minas Gerais - ICMG, \\ Av. Tancredo Neves, 3500, 35170-056 Coronel Fabriciano, M.G., Brazil \\ E. M. A. M. MENDES \\ FUNREI - Fundação de Ensino Superior de São João del Rey, \\ Praça Frei Orlando 170, 36300-000 São João Del Rei, M.G., Brazil
}

Received June 9, 1999; Revised September 6, 1999

\begin{abstract}
This paper investigates the application of discrete nonlinear rational models, a natural extension of the well-known polynomial models. Rational models are discussed in the context of two different problems: reconstruction of chaotic attractors from a time series and the estimation of static nonlinearities from dynamical data. Rational models are obtained via black box identification techniques which only need a relatively short data set. A simple modified algorithm is proposed to handle the noise thus providing a solution to one of the greatest obstacles for estimating rational models from real data. The suggested algorithm and related ideas are tested and discussed using Rössler's equations, real data collected from an implementation of Chua's circuit, logistic map, sine-map with cubic-type nonlinearities, tent map and a map of a feedback buck switching regulator model.
\end{abstract}

\section{Introduction}

In dynamical systems, chaotic behavior is an intrinsically nonlinear phenomenon. A characteristic feature of chaotic systems is an extreme sensitivity to initial conditions while the dynamic motion, at least for dissipative systems, is still constrained to a finite region of the state space called a strange attractor. The physical implication of chaos does not seem to have been widely appreciated until the sixties when the first conclusive demonstration of the existence of chaotic behavior in a simple nonlinear model simulating atmospheric convection was made by Lorenz [1963].

Chaotic behavior has been identified in laboratory experiments such as a water dripping faucet [Crutchfield et al., 1986], simple electric circuits [Briggs, 1987] and nearly turbulent flow such as the Couette-Taylor experiment [Brandstater \& Swinney, 1987]. In practical situations outside laboratory environments, chaotic behavior has been claimed (but not proved in many cases) in medicine [Babloyantz et al., 1985; Roschke \& Basar, 1990a, 1990b; Babloyantz, 1990; Skinner et al., 1990; Freeman, 1990; Govindan et al., 1998], climate records [Nicolis \& Nicolis, 1987; Essex et al., 1987; Ghil et al., 1991], and in several other fields.

Modeling and identification are extremely important steps in the process of developing knowledge about a system. The aim is to search a model or a class of models which best captures the characteristics (dynamical and static) of the system. For instance, models can be used for establishing 
the mechanism of defibrillation of cardiac tissue [Krinsky \& Pumir, 1998] or constructing a differential equation that models the dynamics of a nonlinear series resonant circuit [Hegger et al., 1998].

This paper focuses on the black-box identification of nonlinear systems behaving chaotically which can be seen as a challenging task. Here the choice of the class of nonlinear functions used to represent the system under investigation will be limited to the use of polynomial and rational NARMAX (Nonlinear AutoRegressive Moving Average with eXogenous inputs) models [Chen \& Billings, 1989].

The polynomial structure is very attractive because of the simplicity and the insight it offers of the system properties; therefore they have been extensively used in reproducing nonlinear dynamics. However when the nonlinear functions involved in the system are hard to approximate by polynomial models other approximation schemes should be tried [Aguirre, 1997]. One such scheme, considered as a natural extension of polynomial models, is the rational representation. It has been shown that rational models can represent a wide class of nonlinear dynamics with a few parameters [Billings \& Zhu, 1991]. Both polynomial and rational models have been investigated in detail theoretically and in practical situations. Whereas a number of real examples using polynomial models are available, the same cannot be said for the rational representation.

Insofar as structure detection and parameter estimation are concerned, identification algorithms for rational models are more complex when compared to the ones available for polynomial models. Despite this inherent difficulty, it will be shown that the use of rational models is worthwhile especially in cases where the system under scrutiny exhibits nonlinearities that cannot be modeled by simple polynomial models. Rational models are also compact and very easy to deal with. This is not the case when more complex representations are used.

This paper is organized as follows. In Sec. 2 the nonlinear representation is discussed and a modified identification algorithm is suggested. Section 3 shows models obtained from real data generated from an implementation of Chua's circuit. The same identification procedure is also used for obtaining dynamically valid models for Rössler's equations. In Sec. 4 polynomial and rational models are compared in order to establish the validity of each representation in reconstructing dynamics from real data and recovering static map characteristics. In Sec. 4 examples such as sine-map with cubic-type nonlinearities, tent map and a map for a feedback switching regulator are used to demonstrate that rational discrete models can be used for recovering static characteristics. Finally, Sec. 5 summarizes the main points of the paper.

\section{Background}

\subsection{Nonlinear representations}

Consider the NARMAX model [Leontaritis \& Billings, 1985]

$$
\begin{array}{r}
y(k)=F^{\ell}\left[y(k-1), \ldots, y\left(k-n_{y}\right), u(k-d), \ldots\right. \\
\left.u\left(k-d-n_{u}+1\right), e(k), \ldots e\left(k-n_{e}\right)\right],
\end{array}
$$

where $n_{y}, n_{u}$ and $n_{e}$ are the maximum lags considered for the output, input and noise terms, respectively and $d$ is the delay measured in sampling intervals, $T_{\mathrm{s}}$. Moreover, $u(k)$ and $y(k)$ are respectively the input and output signals. $e(k)$ accounts for uncertainties, possible noise, unmodeled dynamics, etc and $F^{\ell}[\cdot]$ is some nonlinear function of $y(k)$, $u(k)$ and $e(k)$. The function $F^{\ell}[\cdot]$ can, of course, be a polynomial-type function with nonlinearity degree $\ell \in \mathbb{Z}^{+}$. In such a case, to estimate the parameters of this map, Eq. (1) should be expressed in prediction error form as

$$
y(k)=\psi^{\mathrm{T}}(k-1) \hat{\boldsymbol{\theta}}+\xi(k),
$$

where $\psi(k-1)$ is the regressor vector which contains linear and nonlinear combinations of output, input and noise terms up to and including time $k-1$. The parameters corresponding to each regressor are the elements of the vector $\hat{\boldsymbol{\theta}}$. Finally, $\xi(k)$ are the residuals which are defined as the difference between the measured data $y(k)$ and the one-step-ahead prediction $\psi^{\mathrm{T}}(k-1) \hat{\boldsymbol{\theta}}$. The parameter vector $\boldsymbol{\theta}$ can be estimated by orthogonal least-squares techniques [Zhu \& Billings, 1996].

One of the many advantages of such algorithms is that the Error Reduction Ratio (ERR) can be easily obtained as a by-product [Billings et al., 1989; Korenberg et al., 1988], as will be detailed in Sec. 2.3.

Another possibility for the function $F^{\ell}[\cdot]$ in (1) is a rational model that is defined as a ratio 
of two polynomials [Billings \& Chen, 1989]

$$
y(k)=\frac{a\left(y(k-1), \ldots, y\left(k-n_{y}\right), u(k-1), \ldots, u\left(k-n_{u}\right), e(k-1), \ldots, e\left(k-n_{e}\right)\right)}{b\left(y(k-1), \ldots, y\left(k-n_{y}\right), u(k-1), \ldots, u\left(k-n_{u}\right), e(k-1), \ldots, e\left(k-n_{e}\right)\right)}+e(k)
$$

where $u(k)$ and $y(k)$ are as before, $n_{y}, n_{u}$ and $n_{e}$ are the maximum lags of the output, input and noise, respectively. Moreover, such lags need not be the same in the numerator and denominator. $a(k-1)$ and $b(k-1)$ are polynomial functions nonlinear in the regressors taken up to time $k-1$. It is convenient to define the numerator and denominator polynomials in Eq. (3) respectively as [Billings \& Chen, 1989]

$$
\begin{gathered}
a(k-1)=\sum_{j=1}^{N_{\mathrm{n}}} p_{\mathrm{n}} j \boldsymbol{\theta}_{\mathrm{n} j}=\psi_{\mathrm{n}}^{\mathrm{T}}(k-1) \boldsymbol{\theta}_{\mathrm{n}}, \\
b(k-1)=\sum_{j=1}^{N_{\mathrm{d}}} p_{\mathrm{d} j} \boldsymbol{\theta}_{\mathrm{d} j}=\psi_{\mathrm{d}}^{\mathrm{T}}(k-1) \boldsymbol{\theta}_{\mathrm{d}},
\end{gathered}
$$

where $\boldsymbol{\theta}_{\mathrm{n} j}, \boldsymbol{\theta}_{\mathrm{d} j}$ are the parameters of the regressors (of the numerator, $p_{\mathrm{n} j}$, and denominator, $p_{\mathrm{d} j}$,) up to time $k-1$. $N_{\mathrm{n}}+N_{\mathrm{d}}$ is the total number of parameters to be estimated.

The use of Eq. (3) to perform parameter estimation is not straightforward because such a function is nonlinear in the unknown parameters. An alternative solution to this problem is to multiply both sides of Eq. (3) by $b(k-1)$ and rearranging the terms in order to yield [Zhu \& Billings, 1991]

$$
\begin{aligned}
y^{*}(k) & =a(k-1)-y(k) \sum_{j=2}^{N_{\mathrm{d}}} p_{\mathrm{d} j} \theta_{\mathrm{d} j}+b(k-1) e(k) \\
& =\sum_{j=1}^{N_{\mathrm{n}}} p_{\mathrm{n} j} \theta_{\mathrm{n} j}-y(k) \sum_{j=2}^{N_{\mathrm{d}}} p_{\mathrm{d} j} \theta_{\mathrm{d} j}+\zeta(k) \\
& =\psi_{\mathrm{n}}^{\mathrm{T}}(k-1) \boldsymbol{\theta}_{\mathrm{n}}-\psi_{\mathrm{d} 1}^{\mathrm{T}}(k-1) \boldsymbol{\theta}_{\mathrm{d}}+\zeta(k),
\end{aligned}
$$

where $\psi_{\mathrm{d}}^{\mathrm{T}}(k-1)=\left[p_{\mathrm{d} 1} \psi_{\mathrm{d} 1}^{\mathrm{T}}(k-1)\right], \theta_{\mathrm{d} 1}=1$ and

$$
\begin{gathered}
y^{*}(k)=y(k) p_{\mathrm{d} 1}=\frac{a(k-1)}{b(k-1)} p_{\mathrm{d} 1}+p_{\mathrm{d} 1} e(k), \\
\zeta(k)=b(k-1) e(k)=\left(\sum_{j=1}^{N_{\mathrm{d}}} p_{\mathrm{d} j} \theta_{\mathrm{d} j}\right) e(k),
\end{gathered}
$$

where $e(k)$ is white noise. Because $e(k)$ is independent of $b(k-1)$ and has zero mean, it can be written

$$
E[\zeta(k)]=E[b(k-1)] E[e(k)]=0 .
$$

Equation (7) reveals that all the terms of the form $y(k) \psi_{\mathrm{d}}^{\mathrm{T}}(k-1)$, because of $y(k)$, implicitly include the noise $e(k)$ which is correlated with $\zeta(k)$. This, of course, results in parameter bias even if the noise $e(k)$ is white. The aforementioned correlation occurs as a consequence of multiplying (3) by $b(k-1)$ and should be interpreted as the price paid for turning a function which is nonlinear in the parameters to one which is linear in the parameters. It should become clear that both representations, polynomial and rational, will require structure selection, an issue which will be mentioned in the following section.

\subsection{Algorithms}

This section describes the algorithm used to estimate the parameters of rational models. This algorithm is a modification of the one originally proposed in [Billings \& Zhu, 1991]. The present algorithm is a simplified version which assumes that a rational function of the form (3) can be approximated by

$$
\begin{aligned}
y(k)= & \frac{a\left(y(k-1), \ldots, y\left(k-n_{y}\right), u(k-1), \ldots, u\left(k-n_{u}\right)\right)}{b\left(y(k-1), \ldots, y\left(k-n_{y}\right), u(k-1), \ldots, u\left(k-n_{u}\right)\right)} \\
& +c\left(e(k-1), \ldots, e\left(k-n_{e}\right)\right)+e(k),
\end{aligned}
$$


where the noise is modeled as a polynomial, possibly nonlinear. This modification greatly simplifies the original algorithm and in a number of situations has given good results. The basic assumption here is that the regression error can be modeled as possibly nonlinear moving average random process. Hence, the following procedure is suggested.

1. Set $i=0$. Form the regressor matrix and estimate coefficients using ordinary least-squares as

$$
\left[\begin{array}{c}
\hat{\boldsymbol{\theta}}_{\mathrm{n}}^{i} \\
\hat{\boldsymbol{\theta}}_{\mathrm{d} 1}^{i}
\end{array}\right]=\left[\Psi^{\mathrm{T}} \Psi\right]^{-1} \Psi^{\mathrm{T}} \mathbf{y}^{*},
$$

where the superscript $i$ indicates the iteration and the regressor matrix $\Psi \in \mathbb{R}^{N \times N_{\mathrm{n}}+N_{\mathrm{d}}-1}$ is formed taking the regressor vectors $\psi_{\mathrm{n}}(k-1)$ and $\psi_{\mathrm{d} 1}(k-1)$ over the data set $N$, that is

$$
\Psi=\left[\begin{array}{cc}
\psi_{\mathrm{n}}^{\mathrm{T}}(k-1) & \psi_{\mathrm{d} 1}^{\mathrm{T}}(k-1) \\
\vdots & \vdots \\
\psi_{\mathrm{n}}^{\mathrm{T}}(k+N-2) & \psi_{\mathrm{d} 1}^{\mathrm{T}}(k+N-2)
\end{array}\right]
$$

Similarly, $\mathbf{y}^{*} \in \mathbb{R}^{N \times 1}$ is formed taking $y^{*}(k)$ over the data as

$$
\mathbf{y}^{* \mathrm{~T}}=\left[y^{*}(k) y^{*}(k+1) \cdots y^{*}(k+N-1)\right] .
$$

2. Increment counter, $i=i+1$. Calculate the residuals

$$
\xi^{i}(k)=y(k)-\frac{\psi_{\mathrm{n}}^{\mathrm{T}}(k-1) \hat{\boldsymbol{\theta}}_{\mathrm{n}}^{i}}{\psi_{\mathrm{d}}^{\mathrm{T}}(k-1)\left[\begin{array}{c}
1 \\
\hat{\boldsymbol{\theta}}_{\mathrm{d}}^{i}
\end{array}\right]}
$$

and the variance

$$
\left(\sigma_{\xi}^{2}\right)^{i}=\frac{1}{N-m_{\mathrm{d}}} \sum_{i=m_{\mathrm{d}}+1}^{N}\left(\xi^{i}(k)\right)^{2},
$$

where $N$ is the length of the data set and $m_{\mathrm{d}}=$ $\max \left(n_{y}, n_{u}, n_{e}\right)$.

3. Using the residuals computed in step 2, update $\Psi^{\mathrm{T}} \Psi$ and $\Psi^{\mathrm{T}} \mathbf{y}^{*}$ using (16). Also, update (or form for $i=1$ ) the following matrix and vector

$$
\Psi=\left[\begin{array}{ccc}
\psi_{\mathrm{n}}^{\mathrm{T}}(k-1) & y(k) \psi_{\mathrm{d} 1}^{\mathrm{T}}(k-1) & \psi_{\xi}^{\mathrm{T}}(k-1) \\
\vdots & \vdots & \vdots \\
\psi_{\mathrm{n}}^{\mathrm{T}}(k+N-2) & y(k) \psi_{\mathrm{d} 1}^{\mathrm{T}}(k+N-2) & \psi_{\xi}^{\mathrm{T}}(k+N-2)
\end{array}\right]
$$

where $\psi_{\xi}$ are regressors of the noise model. Because the noise is not measured, the residuals determined in step 2 are used instead. It is interesting to note that the matrix elements $\sum_{k=1}^{N} p_{\mathrm{d} i} p_{\mathrm{d} j}$ are approximations of $N r$, where $r$ is the correlation coefficient between regressor $p_{\mathrm{d} i}$ and $p_{\mathrm{d} j}$.

4. Determine

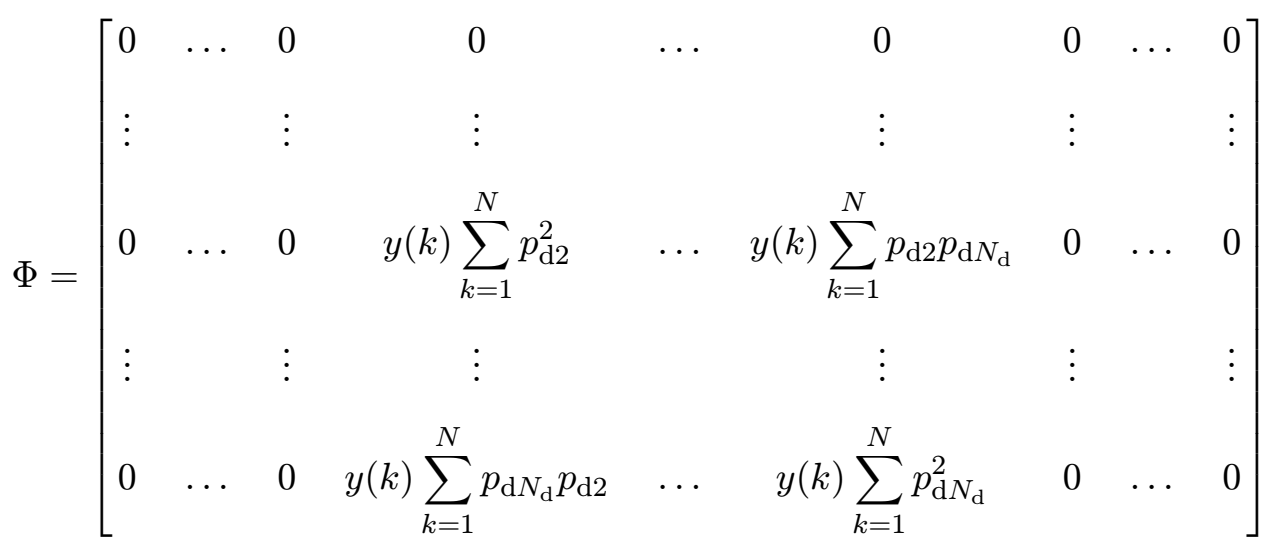


and

$$
\phi=\left[\begin{array}{c}
0 \\
\vdots \\
0 \\
-y(k) \sum_{k=1}^{N} p_{\mathrm{d} 2} p_{\mathrm{d} 1} \\
N(k) \sum_{k=1}^{N} p_{\mathrm{d} N_{\mathrm{d}}} p_{\mathrm{d} 1} \\
0 \\
\vdots \\
0
\end{array}\right]
$$

and re-estimate the parameters using

$$
\left[\begin{array}{c}
\hat{\boldsymbol{\theta}}_{\mathrm{n}}^{i} \\
\hat{\boldsymbol{\theta}}_{\mathrm{d} 1}^{i}
\end{array}\right]=\left[\Psi^{\mathrm{T}} \Psi-\left(\xi_{\xi}^{2}\right)^{i} \Phi\right]^{-1}\left[\Psi^{\mathrm{T}} \mathbf{y}^{*}-\left(\xi_{\xi}^{2}\right)^{i} \phi\right] .
$$

5. Return to step 2 until convergence (of parameters or residual variance).

The procedure outlined above is simple to understand but inadequate for implementation because equations such as (11) and (19) will be typically ill-conditioned. Also, step 1 assumes that the regressors have been previously chosen. In practice these two difficulties are dealt with simultaneously using orthogonal techniques. In particular, structure selection in step 1 is carried out using the Error Reduction Ratio (ERR) criterion [Billings \& Chen, 1989], as detailed in the following section.

\subsection{Forward selection using orthogonalization}

To avoid an excessive number of parameters in Eq. (6), an efficient subset selection procedure can be performed, based on the orthogonal least squares (OLS) method [Billings et al., 1989]. Given the full set of candidate process terms (regressors) that are selected one by one such that at each stage the chosen term results in the best fit for the estimation data from the remaining set of regressors. This does not guarantee that the best subset model will be found. Numerous applications of the OLS algorithm in real problems however demonstrate that this is not a major drawback and the subset model found is usually very good.

The columns of $\Psi$ are indicated by $p_{i}$ and each column represents a regressor. Briefly, the basic principle of the orthogonal estimator is to replace the original set of regression vectors $p_{i}$ (also called basis vectors) for a set of orthogonal vectors. The parameters associated with new vectors are such that the contribution of each vector can be measured independently of the rest of the vectors. This makes possible the selection of relevant terms in a rational model.

The regression vector $\left[p_{1} \ldots p_{N_{\mathrm{n}}+N_{\mathrm{d}}-1}\right]^{\mathrm{T}}$ forms a set of basis vectors, and the OLS solution, $\hat{\boldsymbol{\theta}}$, satisfies the condition that $\Psi \hat{\boldsymbol{\theta}}$ will be the projection of $\mathbf{y}^{*}$ onto the space spanned by these basis vectors. The OLS method involves the transformation of the set of original vectors spanned by $p_{i}$ into a set of orthogonal basis vectors, and thus makes it possible to calculate the individual contribution to the desired output from each basis vector.

The regression matrix $\Psi$ can be decomposed into $\Psi=Q R$, where $R$, shown below, is an $\left(N_{\mathrm{n}}+N_{\mathrm{d}}-1\right) \times\left(N_{\mathrm{n}}+N_{\mathrm{d}}-1\right)$ triangular matrix with 1's on the diagonal and 0's below and $Q$ is an $N \times\left(N_{\mathrm{n}}+N_{\mathrm{d}}-1\right)$ matrix with orthogonal columns $q_{i}$ such that $Q^{\mathrm{T}} Q=D$, where $D$ is a diagonal matrix.

Since the space spanned by the set of orthogonal basis vectors $q_{i}$ is the same space spanned by the original set of vectors $\left(p_{i}\right)$, the regression equation (6) gives rise to the following matrix equation over the data set

$$
\mathbf{y}^{*}=Q \hat{\mathbf{g}}+\boldsymbol{\xi}
$$

where $\boldsymbol{\xi}$ is the equation error vector. The OLS solution, $\hat{\mathbf{g}}$, can be calculated using

$$
\begin{gathered}
\hat{\mathrm{g}}=D^{-1} Q^{\mathrm{T}} \mathbf{y}^{*} \quad \text { or } \quad \hat{g}_{i}=\frac{q_{i}^{\mathrm{T}} \mathbf{y}^{*}}{q_{i}^{\mathrm{T}} q_{i}}, \\
1 \leq i \leq\left(N_{\mathrm{n}}+N_{\mathrm{d}}-1\right) .
\end{gathered}
$$

The original set of parameters $\hat{\boldsymbol{\theta}}$ can be retrieved by solving the triangular system $R \hat{\boldsymbol{\theta}}=\hat{\mathrm{g}}$.

A great advantage of the orthogonal estimator is the possibility of selecting the relevant vectors (terms) as a by-product. To demonstrate this, consider again the orthogonal regression equation (20). 
In doing so, it is assumed that the orthogonal property $q_{i}^{\mathrm{T}} q_{j}=0$ for $i \neq j$ holds. Therefore, if Eq. (20) is multiplied by itself and the time average is taken, the following equation can be derived

$$
\frac{1}{N} \mathbf{y}^{* \mathrm{~T}} \mathbf{y}^{*}=\frac{1}{N} \sum_{i=1}^{\left(N_{\mathrm{n}}+N_{\mathrm{d}}-1\right)} \hat{g}_{i}^{2} q_{i}^{\mathrm{T}} q_{i}+\frac{1}{N} \boldsymbol{\xi}^{\mathrm{T}} \boldsymbol{\xi} .
$$

The output mean square value (MSV) $\mathbf{y}^{* \mathrm{~T}} \mathbf{y}^{*} N$ consists of two terms. The first term, $\sum_{i=1}^{\left(N_{\mathrm{n}}+N_{\mathrm{d}}-1\right)} \hat{g}_{i}^{2} q_{i}^{\mathrm{T}} q_{i} / N$, is the part of the output MSV explained by the regressors whereas the second term, $\boldsymbol{\xi}^{\mathrm{T}} \boldsymbol{\xi} / N$, accounts for the unexplained output MSV. Owing to the orthogonal estimator, the increment towards the overall output MSV of each regressor (term or vector) can be computed independently as $\hat{g}_{i}^{2} q_{i}^{\mathrm{T}} q_{i}$. Expressing this quantity as a fraction of the overall output MSV yields the Error Reduction Error (ERR)

$$
[E R R]_{i}=\frac{\hat{g}_{i}^{2} q_{i}^{\mathrm{T}} q_{i}}{\mathbf{y}^{* \mathrm{~T}} \mathbf{y}^{*}}, \quad 1 \leq i \leq\left(N_{\mathrm{n}}+N_{\mathrm{d}}-1\right),
$$

that can be used as a simple and effective means of selecting the most relevant regressors in a forwardregression manner. Therefore ERR imposes a hierarchy of terms according to their contribution towards the overall output MSV. In the actual implementation of the algorithm, the parameters need not be estimated in order to determine the respective ERR value. Hence, (23) can be implemented with $\hat{g}_{i}$ as given in (21).

\section{Results}

The main goal of this paper is the reconstruction of equations which would successfully describe the system motion. The approach used here is to fit rational models using the identification algorithm described above. No pre-processing of the data was performed. As can be seen in the remainder of the paper, the identified models will reproduce the underlying system characteristics and are extremely compact. As a last remark, it is pointed out that because all the benchmarks used, including the real implementation of Chua's circuit, are autonomous systems, the identified models are, strictly speaking, NARMA models. This means that the regressor vectors $\psi_{\mathrm{n}}(k-1)$ and $\psi_{\mathrm{d}}(k-1)$ do not have exogenous regressors of the form $u(k-i)$.

\subsection{A practical implementation of Chua's circuit}

Chua's circuit is shown in Fig. 1. The only nonlinear element is the two-terminal piecewise-linear resistor denoted "Chua's diode". This nonlinear element can be easily implemented using "offthe-shelf" components as suggested in [Kennedy, 1992]. The measured current-voltage characteristic of Chua's diode is shown in Fig. 2. The equations governing the circuit dynamics can be obtained by inspection and are

$$
\left\{\begin{array}{l}
C_{1} \frac{d v_{1}}{d t}=\frac{\left(v_{2}-v_{1}\right)}{R}-i_{\mathrm{d}}\left(v_{1}\right) \\
C_{2} \frac{d v_{2}}{d t}=\frac{\left(v_{1}-v_{2}\right)}{R}+i_{L} \\
L \frac{d i_{L}}{d t}=-v_{2}-r_{L} i_{L}
\end{array}\right.
$$

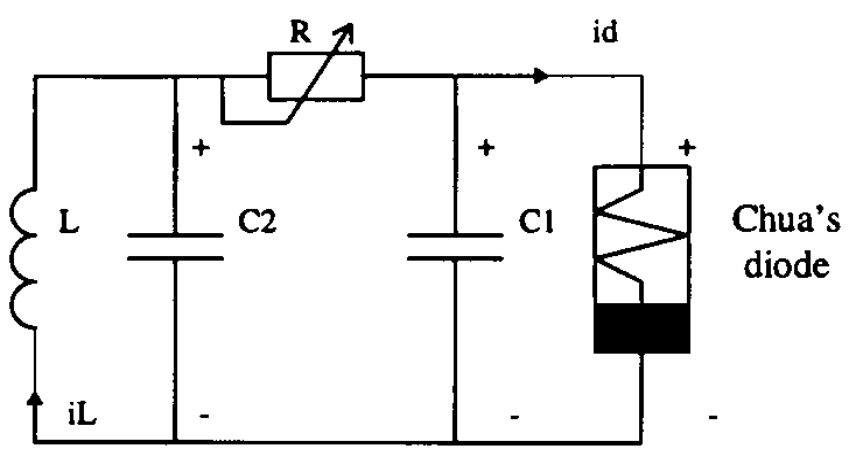

Fig. 1. Chua's circuit.

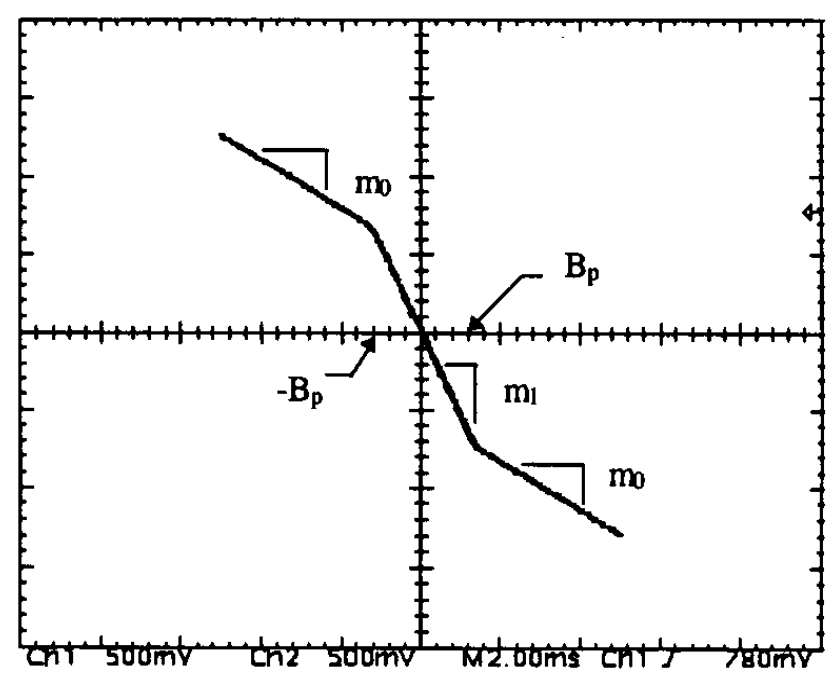

Fig. 2. Measured voltage versus current characteristic of Chua's diode, the only nonlinear element in the circuit. 


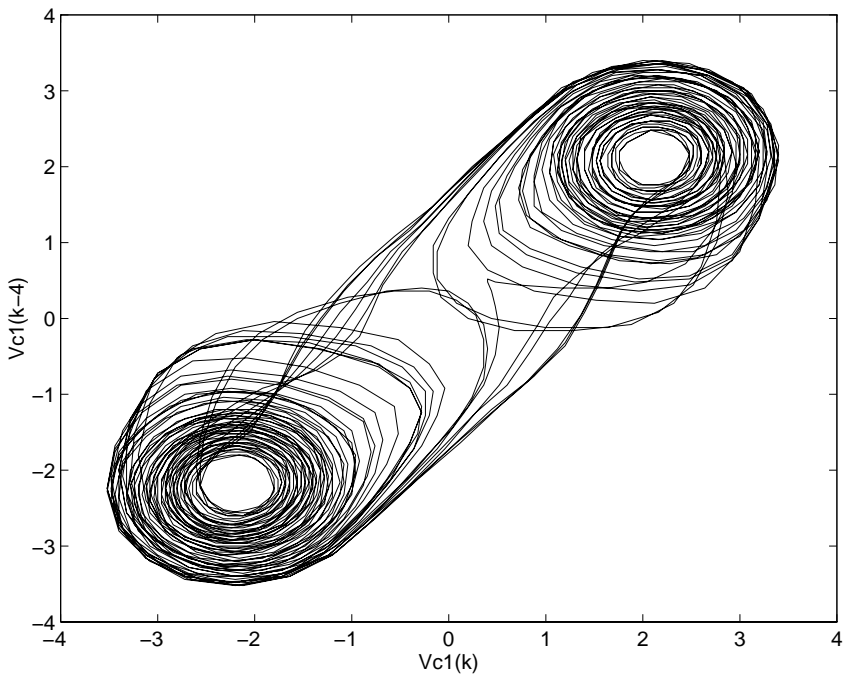

Fig. 3. Bidimensional reconstruction (2000 points) of Chua's double-scroll attractor. A window of one thousand points of these data were used in the identification.

where $v_{i}$ is the voltage across capacitor $C_{i}, i_{L}$ is the current through the inductor and the current through Chua's diode is given by

$$
i_{\mathrm{d}}\left(v_{1}\right)=\left\{\begin{array}{ll}
m_{0} v_{1}+B_{\mathrm{p}}\left(m_{0}-m_{1}\right) & v_{1}<-B_{\mathrm{p}} \\
m_{1} v_{1} & \left|v_{1}\right| \leq B_{\mathrm{p}} \\
m_{0} v_{1}+B_{\mathrm{p}}\left(m_{1}-m_{0}\right) & v_{1}>-B_{\mathrm{p}}
\end{array},\right.
$$

where $B_{\mathrm{p}}, m_{0}$ and $m_{1}$ are respectively the break point and the inclinations of the piecewise-linear function shown in Fig. 2.

The following components were used: $C_{1}=$ $10 \pm 0.5 \mathrm{nF}, C_{2}=90 \pm 5 \mathrm{nF}, L=21 \pm 2 \% \mathrm{mH}$, $r_{L}=15 \Omega$ and $R$ is a $2.0 \mathrm{k} \Omega$ trimpot, $m_{0}=$ $-0.37 \pm 0.04 \mathrm{mS}, m_{1}=-0.68 \pm 0.04 \mathrm{mS}$ and $B_{\mathrm{p}}=1.1 \pm 0.2 \mathrm{~V}$.

Varying the trimpot $R$, the dynamics of this circuit settle to several different regular and chaotic attractors such as the double-scroll attractor attained with $R \approx 1800 \Omega$ and the spiral attractor which is observed when $R \approx 1900 \Omega$. Figure 3 shows the bidimensional attractors reconstructed using the measured data with estimated signal to noise ratio $\left(20 \log \sigma^{2}\right.$ (signal) $/ \sigma^{2}$ (noise)) of approximately $72.3 \mathrm{~dB}$. Such data were sampled at $T_{\mathrm{s}}=12 \mu \mathrm{s}$ and 5000 samples were recorded with a resolution of 12 bits. No anti-aliasing analog filtering was performed, and the choice of the sampling time was made based upon correlation functions [Aguirre et al., 1997].

\subsubsection{Polynomial and rational models for Chua's circuit}

In this section one thousand data points of the output $y(k)$, the voltage over capacitor $C_{1}$, were used in the identification. The following polynomial model was obtained [Rodrigues, 1996]:

$$
\begin{aligned}
y(k)= & 3.5230 y(k-1)-4.287 y(k-2)-0.2588 y(k-4)-1.7784 y(k-1)^{3}+2.0652 y(k-3) \\
& +6.1761 y(k-1)^{2} y(k-2)+0.1623 y(k-1) y(k-2) y(k-4)-2.7381 y(k-1)^{2} y(k-3) \\
& -5.5369 y(k-1) y(k-2)^{2}+0.1031 y(k-2)^{3}+0.4623 y(k-4)^{3}-0.5247 y(k-2)^{2} y(k-4) \\
& -1.8965 y(k-1) y(k-3)^{2}+5.4255 y(k-1) y(k-2) y(k-3)+0.7258 y(k-2) y(k-4)^{2} \\
& -1.7684 y(k-4)^{2} y(k-3)+1.1800 y(k-4) y(k-3)^{2}+\psi_{\xi}^{\mathrm{T}}(k-1) \hat{\boldsymbol{\theta}}_{\xi}+\xi(k),
\end{aligned}
$$

where $\psi_{\xi}^{\mathrm{T}}(k-1) \hat{\boldsymbol{\theta}}_{\xi}$ is the noise model composed of 20 linear terms of the form $\xi(k-j)$ used to avoid bias. This part of the model was not used in the simulations shown below. The identification of model (26) and the cluster analysis of the double-scroll and spiral attractors have been discussed in detail in [Aguirre et al., 1997].

Using the same data as described above and also term clustering information [Mendes, 1995; Aguirre et al., 1997; Aguirre \& Jácome, 1998], the following rational model was obtained [Corrêa, 1997]

$$
\begin{aligned}
y(k)= & \frac{1}{D} \times\left(2.5568 y(k-1)-1.7594 y(k-2)+0.2696 y(k-5)+0.6192 y(k-1)^{3}-1.0219 y(k-2)^{3}\right. \\
& -3.2455 y(k-1)^{2} y(k-5)+0.0735 y(k-3)^{3}+0.3444 y(k-1) y(k-5)^{2} \\
& \left.-0.4401 y(k-2) y(k-5)^{2}+3.4624 y(k-1) y(k-2) y(k-5)+0.1986 y(k-1)^{2} y(k-2)\right) \\
& +\sum_{i=1}^{10} \hat{\theta}_{i} \xi(k-i)+\sum_{j=1}^{5} \hat{\theta}_{j} \xi(k-j)^{2}+\xi(k)
\end{aligned}
$$


where

$$
\begin{aligned}
D= & 1+1.5164 y(k-1) y(k-2)+0.5657 y(k-3) y(k-5)-12.8527 y(k-2)^{2}+0.1948 y(k-1)^{2} \\
& +1.7662 y(k-2) y(k-5)-0.1409 y(k-5)^{2}-2.0470 y(k-1) y(k-5) .
\end{aligned}
$$

Figure 4 shows the chaotic double-scroll attractors reconstructed using polynomial model (26) and rational model (27). Table 1 shows the estimated fixed points and largest Lyapunov exponents for both the original system and the identified models.

The results suggest that both polynomial and rational models do reproduce the characteristic dynamical features of the original attractor. Such results also seem to indicate that the polynomial representation is slightly better as far as the modeling of attractors produced by Chua's circuit is concerned. This should come as no surprise because, in fact, the set of equations which describe Chua's circuit can be expressed in polynomial form. On the other hand, in cases where the system has a static nonlinearity with relatively "sharp edges" the use of rational models might be advantageous, especially if the original system is autonomous [Aguirre, 1997]. This will be demonstrated in the next sections.

\subsection{Rössler's Equations}

Rössler's equations is another benchmark widely used to study chaotic dynamics and can be represented as follows [Rössler, 1076]:

$$
\left\{\begin{array}{l}
\dot{x}=-y-z, \\
\dot{y}=x+a y \\
\dot{z}=b+z(x-c) .
\end{array}\right.
$$

As demonstrated in [Gouesbet \& Letellier, 1994], the reconstruction of the attractor through the observation of a single variable $x$ or $z$ requires a rational representation. This fact happens due to the product of $x$ and $z$ that appears in the third dynamic equation of the system. On the other hand, reconstruction from measurements of the $y$ variable would be typically polynomial.

In what follows polynomial and rational models identified from the variable $x$ in Rossler's equations are listed:

$$
\begin{aligned}
x(k)= & +0.1972 \times 10 x(k-1)-0.104 \times 10 x(k-2)+0.7456 \times 10^{-4} x(k-4) x(k-2)^{3} x(k-1) \\
& -0.2053 \times 10^{-4} x(k-5) x(k-4)^{4}-0.285 \times 10^{-4} x(k-5) x(k-1)^{4} \\
& +0.2484 \times 10^{-4} x(k-3)^{2} x(k-2)^{3}+0.1238 \times 10^{-2} x(k-2) x(k-1)^{2}+0.4353 \times 10^{-4} x(k-5)^{4} \\
& +0.2258 \times 10^{-2} x(k-5) x(k-2) x(k-1)^{2}+0.3123 \times 10^{-4} x(k-4)^{5}+0.7531 \times 10^{-3} x(k-1)^{4} \\
& -0.2703 \times 10^{-2} x(k-3)^{2} x(k-1)^{2}-0.7807 \times 10^{-3} x(k-1)^{3} \\
& -0.7077 \times 10^{-4} x(k-3)^{2} x(k-2)^{2} x(k-1)-0.3304 \times 10^{-3} x(k-3) x(k-2)^{3} \\
& -0.8847 \times 10^{-2} x(k-5) x(k-1)+0.7631 \times 10^{-2} x(k-4) x(k-1) \\
& -0.387 \times 10^{-4} x(k-5)^{3} x(k-1)^{2}+0.4676 \times 10^{-3} x(k-3)^{3} x(k-1) . \\
& \\
& \frac{1}{D} \times\left\{+0.2718 \times 10 x(k-1)-0.2545 \times 10 x(k-2)-0.7374 \times 10^{-2} x(k-1) x(k-2) x(k-4)\right. \\
& +0.3883 \times 10^{-2} x(k-4)^{3}+0.7798 \times 10^{-2} x(k-2) x(k-3) x(k-4)+0.8335 x(k-3) \\
& -0.2614 \times 10^{-1} x(k-5)+0.9227 \times 10^{-3} x(k-2)^{2} x(k-4) \\
& -0.6736 \times 10^{-2} x(k-1)^{2} x(k-4)-0.3483 \times 10^{-2} x(k-4)^{2} x(k-5) \\
& \left.+0.6479 \times 10^{-3} x(k-4) x(k-5)-0.2461 \times 10^{-2} x(k-3) x(k-4)^{2}\right\}
\end{aligned}
$$


Table 1. Dynamical invariants of data and identified models for Chua's Circuit.

\begin{tabular}{cccc}
\hline & Data & Polynomial (26) & Rational (27)-(28) \\
\hline Fixed Points & $(0,2.24,-2.24)$ & $(0,2.24,-2.24)$ & $(0,2.37,-2.37)$ \\
Larg. Lyapunov Exp. & $1.3516 \pm 0.0343$ & $1.3350 \pm 0.0563$ & $1.4218 \pm 0.0596$ \\
\hline
\end{tabular}

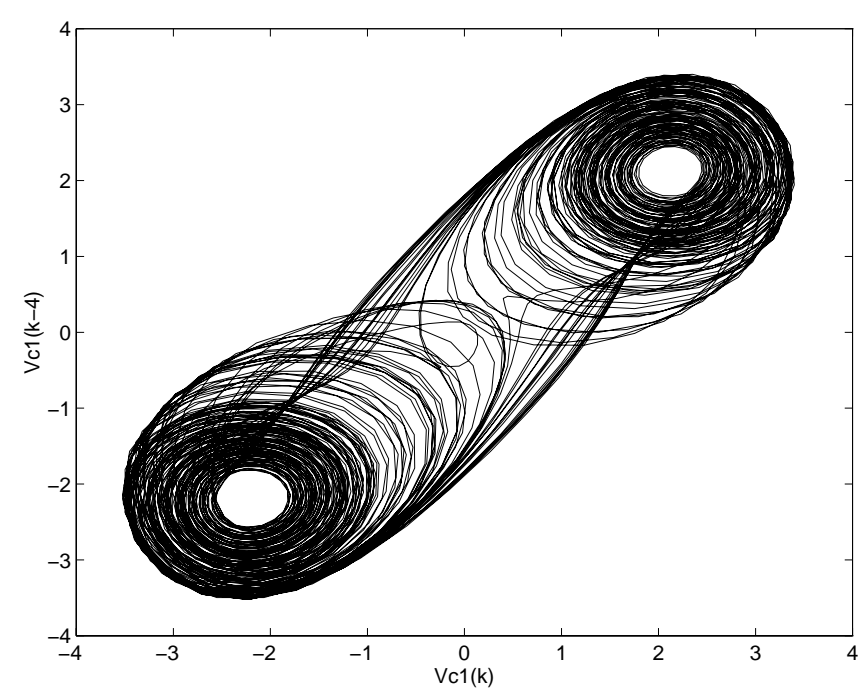

(a)

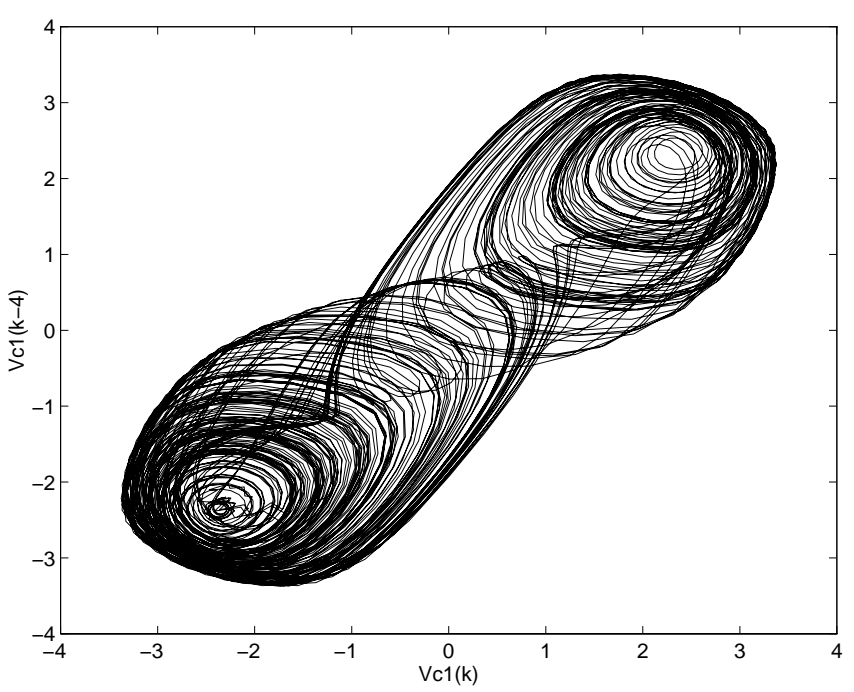

(b)

Fig. 4. Double-scroll attractors reconstructed from (a) simulation of the polynomial model (26) and (b) simulation of the rational model (27). The $x$-axis is $y(k)$ and the $y$-axis is $y(k-4)$.

where

$$
\begin{aligned}
D= & 1-0.742 \times 10^{-2} x(k-1) x(k-4) \\
& +0.412 \times 10^{-5} x(k-1)^{3} \\
& +0.1515 \times 10^{-4} x(k-1) x(k-4) x(k-5) .
\end{aligned}
$$

Alike model (27), a noise model was estimated (but not shown) with models (30) and (31) to reduce bias. For the sake of simplicity the noise models henceforth will be omitted. As can be seen, both models have the same maximum lag, ${ }^{1}$ that is, 5 and are therefore of the same order. However it can be noticed that the degree of nonlinearity of the rational model is smaller, as well as the polynomials composing number of process terms.

Figure 5 presents bidimensional projection of Rössler's equations reconstructed using the $x$ variable of the original system and also using models (30) and (31). The fact that the attractor reconstructed from the rational model looks more similar to the original one is due to the fact that the struc- ture of such a model is a more "natural" representation to approximate the Rössler attractor from the $x$ variable. This does not automatically guarantee that the reconstructed attractor using model (31) is valid nor disqualifies model (30). To do this it would be necessary to verify if the reconstructed models are topologically equivalent to the original attractor [Gilmore, 1998; Letellier et al., 1995]. From Table 2 it seems appropriate to infer that the rational model is able to represent the dynamics of Rössler's equations with less parameters and a smaller degree of nonlinearity. This reinforces the previous remark that the flow reconstructed from the $x$ variable is typically rational.

\section{Recovering Maps Static Nonlinearities}

An interesting tool when the objective is to validate and select the structure of identified models is the estimation of static nonlinearities [Aguirre, 1997].

\footnotetext{
${ }^{1}$ Henceforth denoted order or dimension. The order, degree of nonlinearity and number of terms are thoroughly discussed and analyzed in [Mendes \& Billings, 1998].
} 


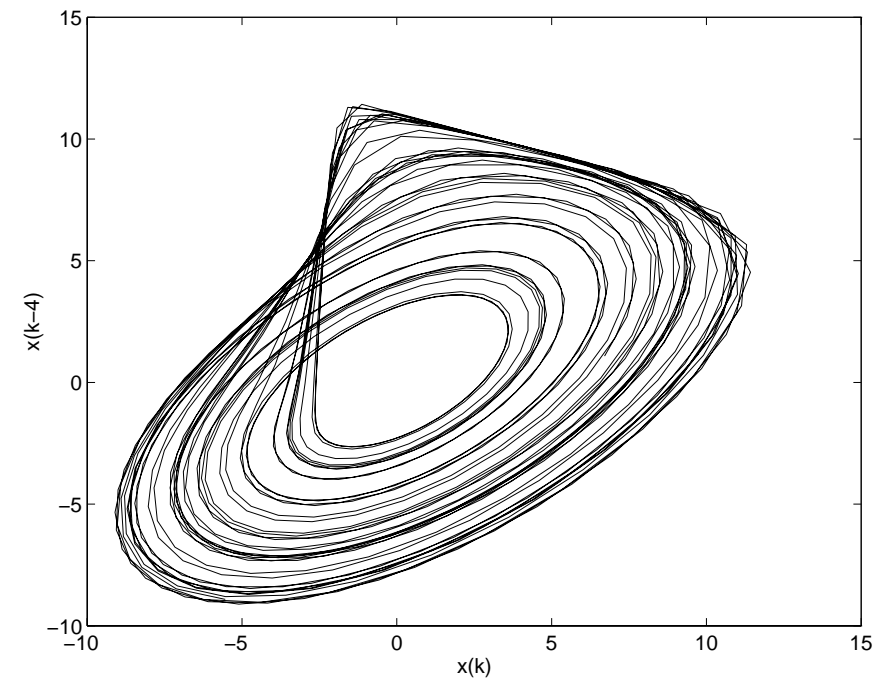

(a)

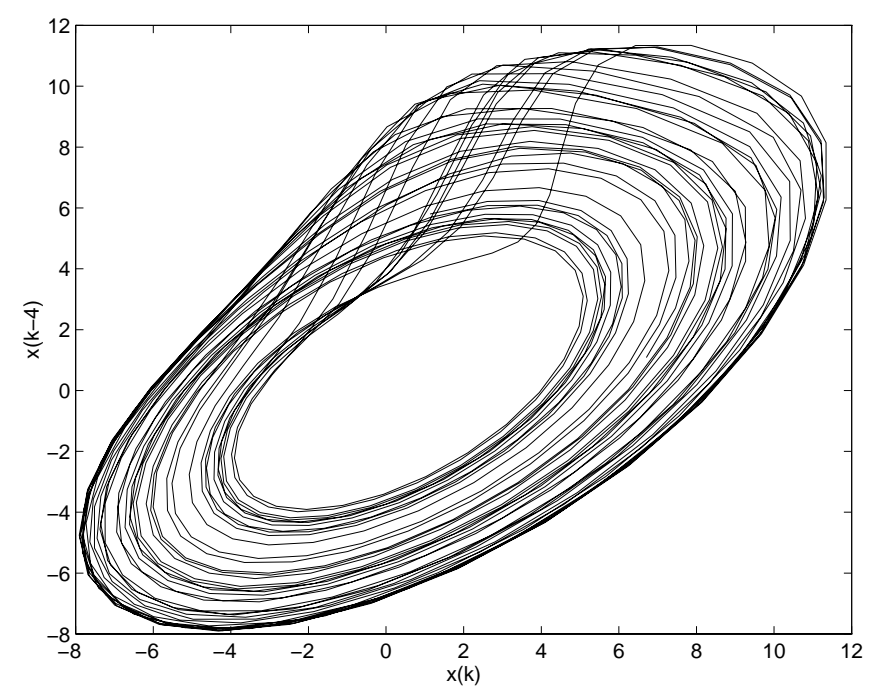

(b)

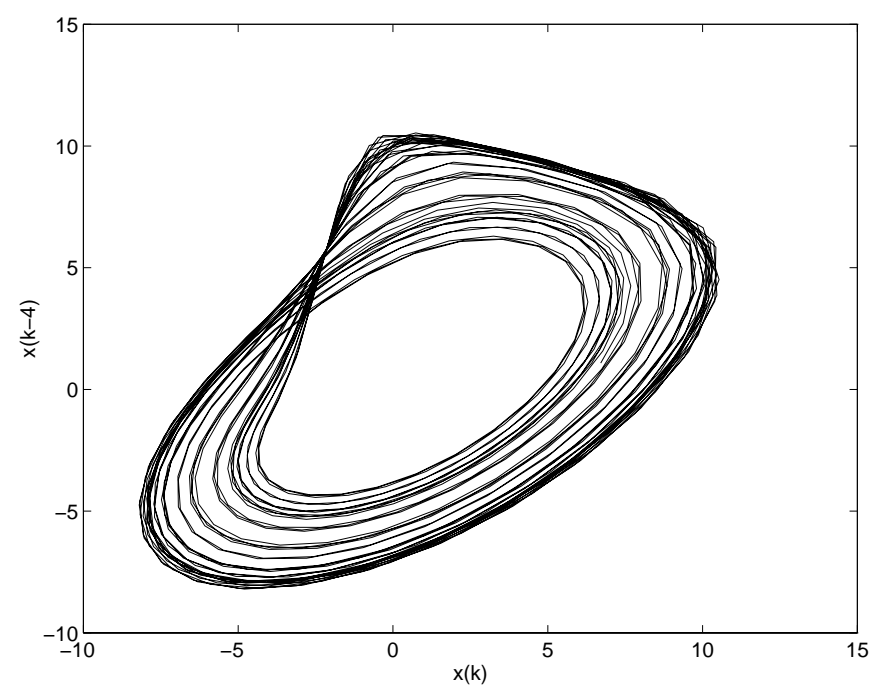

(c)

Fig. 5. Bidimensional projection of $x$ variable for Rössler system, using: (a) simulated data (b) polynomial model (c) rational model.

In this section a study on the reconstruction of first return maps using rational models is presented. The results will be discussed in the light of examples such as the sine-map with cubictype nonlinearities; tent map and a map of a feedback buck switching regulator model. It is desired to obtain dynamically valid models which also correctly represent the static nonlinearity of the system. The reconstruction of first return maps using continuous-time models is being investigated by Letellier and co-workers [Ménard et al., 1999].

\subsection{Sine-map with cubic-type nonlinearities}

Consider the following map

$$
y(k)=\alpha \sin (y(k-1)),
$$

where $\alpha=\pi$ and initial condition $y(0) \in[0, \pi]$.

Using 500 points generated by the simulation of Eq. (33), the following polynomial [Aguirre, 1997] and rational models were obtained

$$
y(k)=+0.29893 \times 10 y(k-1)-0.2479 y(k-1)^{3}
$$


Table 2. Dynamical invariants of data and identified models for Rössler system.

\begin{tabular}{cccc}
\hline & Data & Polynomial & Rational \\
\hline Fixed Points & $(0.0070,5.6930)$ & $(0,21.5425$, & $(0,-3.9033 \pm 9.5741 \mathrm{i}$, \\
& & $3.4002 \pm 11.4882 \mathrm{i},-10.8118)$ & $9.3869)$ \\
Larg. Lyapunov Exp. & $1.2418 \pm 0.3987$ & $1.5661 \pm 0.2814$ & $0.6118 \pm 0.1607$ \\
\hline
\end{tabular}

Table 3. Dynamical invariants of data and identified models for Sine-map with cubic nonlinearities.

\begin{tabular}{cccc}
\hline & Data & Polynomial Model & Rational Model \\
\hline Fixed Points & $(2.439,0,-2.439)$ & $(2.610,0,-2.610)$ & $(2.400,0,-2.400)$ \\
Larg. Lyapunov Exp. & $1.1574 \pm 0.1105$ & $1.0544 \pm 0.0883$ & $1,1544 \pm 0.0077$ \\
\hline
\end{tabular}

and

$y(k)=\frac{1}{D} \times\left\{+0.3957 \times 10 y(k-1)-0.3951 y(k-1)^{3}\right\}$,

where

$$
\begin{aligned}
D= & 1-0.5117 \times 10^{-3} y(k-2)^{2} \\
& -0.3722 \times 10^{-2} y(k-3)^{2} \\
& +0.8053 \times 10^{-2} y(k-1) y(k-2) \\
& -0.2728 \times 10^{-2} y(k-1) y(k-3) \\
& +0.1174 y(k-1)^{2} .
\end{aligned}
$$

It is observed that Eq. (34) is a first-order polynomial model with two process terms, whereas Eq. (35) is a rational model with eight process terms. However note in Fig. 6 that the static map reconstructed from the rational model is more accurate than the polynomial counterpart.

Table 3 shows the fixed points and the Lyapunov exponents of the data and models. Clearly, the rational model reproduces the original system characteristics better than the polynomial one.

\subsection{Tent map}

Consider now the tent map described by

$$
y(k)=1-1.999|y(k-1)-0.5| .
$$

A close look at Fig. 7 demonstrates that, despite the apparent polynomial structure of Eq. (37), the rational representation is more efficient to reproduce the original static nonlinearity. One

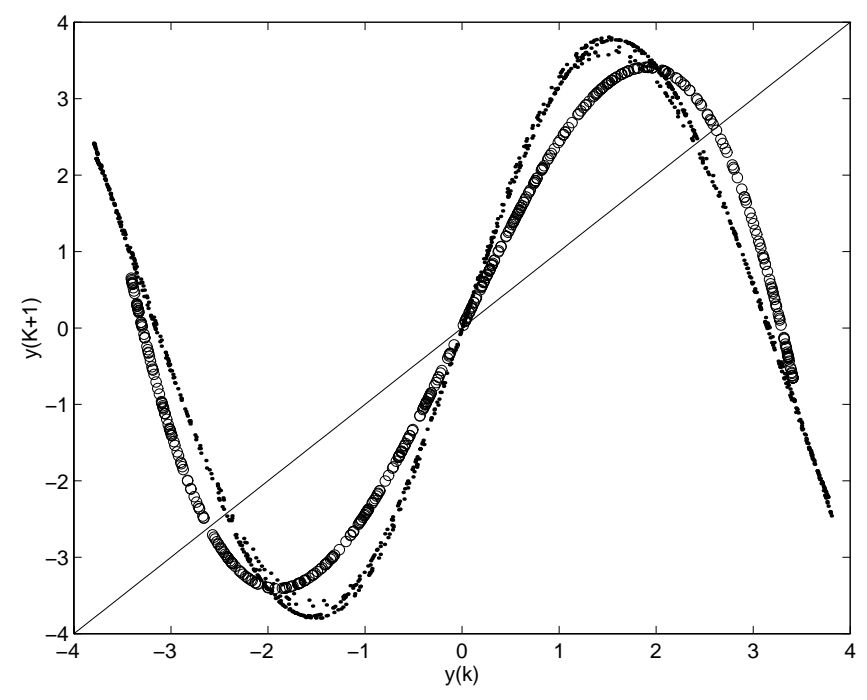

Fig. 6. (.) Original and rational model of embedded attractor, (o) polynomial model. (-) is the $y=x$ line. The static nonlinearity estimated with the rational model is the noisier curve indicated with dots.

identified model that can reproduce the tent map quite well is

$$
\begin{aligned}
y(k)= & \frac{1}{D} \times\left\{+0.2608 \times 10^{-1}\right. \\
& -0.1325 \times 10 y(k-1) y(k-1) \\
& +0.1325 \times 10 y(k-1)\}
\end{aligned}
$$

where

$$
\begin{aligned}
D= & 1-0.2416 \times 10 y(k-1) \\
& +0.2407 \times 10 y(k-1) y(k-1) .
\end{aligned}
$$




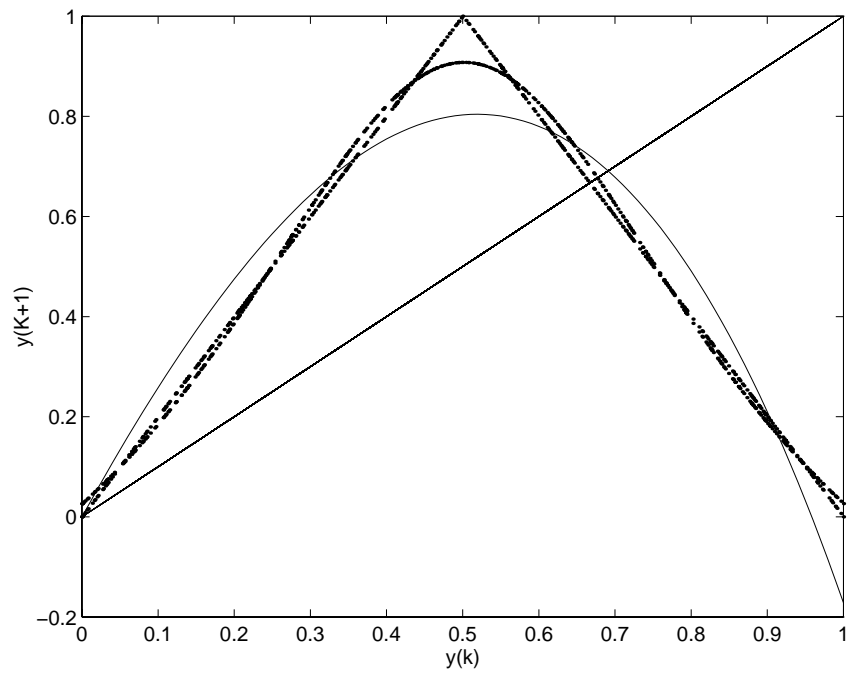

Fig. 7. 500 data points on the tent attractor (sharp edge). Static nonlinearities estimated using $(\cdot)$ the rational model (38) and (-) a polynomial model with three terms and cubic degree of nonlinearity [Aguirre, 1997]. The straight line is $y=x$.

\subsection{First return map of a feedback buck switching regulator model}

In this section a rational model is estimated for a feedback buck switching regulator model. This regulator operates in closed-loop to guarantee a certain voltage in the load [Tse, 1994]. The map obtained from the circuit equations has the following general form [Tse, 1994]:

$$
\begin{aligned}
y(k)= & \alpha y(k-1) \\
& +\frac{h\left(d_{n}\right)^{2} \beta E[E-y(k-1)]}{y(k-1)}
\end{aligned}
$$

where $\alpha=0.8872, \beta=1.2$ and $E=33$ are constants which only depend on the circuit components, $d_{n}$ is the controller output (a voltage signal) and the saturation $h\left(d_{n}\right)$ is given by

$$
h\left(d_{n}\right)= \begin{cases}0 & \text { if } d_{n}<0 \\ 1 & \text { if } d_{n}>1 \\ d_{n} & \text { otherwise }\end{cases}
$$

In [Aguirre, 1997] it is shown that polynomial models are unable to reproduce the dynamics of Eq. (40). This is not the case when rational models are identified using the same data set as it will be demonstrated below.

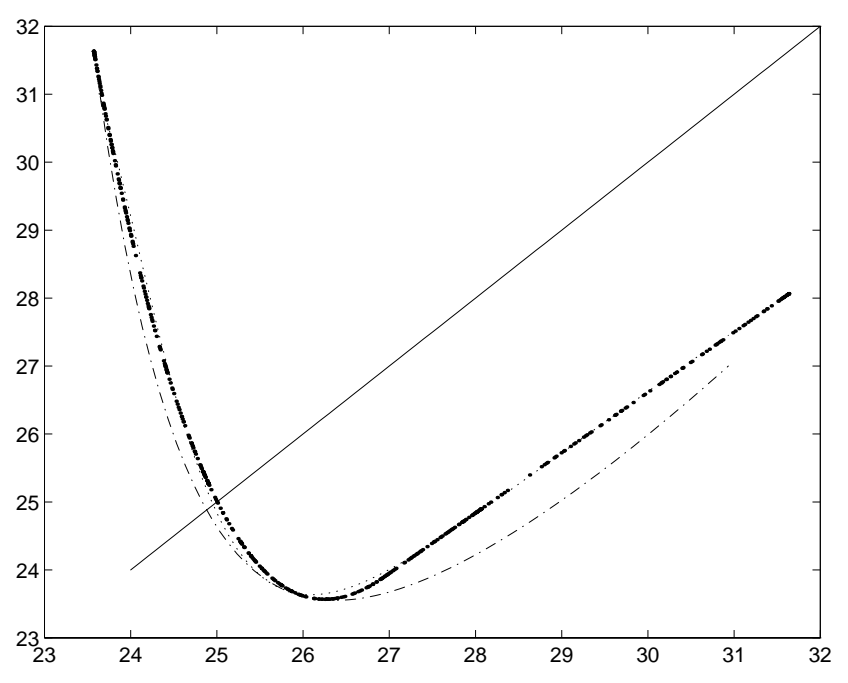

Fig. 8. First return maps for a buck regulator model. Heavy dots are data, light dots correspond to the rational model (42), dash-dot corresponds to an ad hoc model in [Aguirre, 1997] and solid is the line $x=y$.

The rational model below reproduces the dynamics of Eq. (40)

$$
\begin{aligned}
y(k)= & \frac{1}{D} \times\{+0.8658 \times 10 \\
& +0.1223 \times 10^{-2} y(k-1)^{3} \\
& \left.-0.441 \times 10^{-1} y(k-1)^{2}\right\},
\end{aligned}
$$

where

$$
\begin{aligned}
D= & 1-0.8381 \times 10^{-1} y(k-1) \\
& +0.1766 \times 10^{-2} y(k-1)^{2} .
\end{aligned}
$$

In Fig. 8 the first return map reconstructed using the original data and by iterating the rational model in Eq. (42) is depicted. Note that the rational model can approximate the original static nonlinearity better than the ad hoc model obtained in [Aguirre, 1997].

Fixed points and Lyapunov exponents calculated using model (42) confirm that such a model is indeed a good dynamical approximation of the original system characteristics. The results are displayed in Table 4.

\section{Conclusions}

In this work it has been shown that rational models can be used for modeling complex dynamics. The use of such models are well justified when polynomial models cannot approximate the original static characteristics and dynamics of the system under 
Table 4. Validation of estimated models for buck switching regulator.

\begin{tabular}{ccc}
\hline & Data & Rational Model \\
\hline Fixed Point & $(25.0018)$ & $(24.9561)$ \\
Larg. Lyapunov Exp. & $0.4422 \pm 0.2054$ & $0.4212 \pm 0.1747$ \\
\hline
\end{tabular}

investigation. Despite being more complex than polynomial models, rational models are compact and easy to deal with. However it is worth mentioning that their structure needs careful selection. A modified algorithm has been proposed to circumvent this problem. The use of rational models to model chaotic dynamics is in no way different from the use of such a representation to model nonlinear regular dynamics. In the present work emphasis has been given to the problem of modeling chaos (especially in Sec. 4) because a chaotic time series usually has sufficient dynamical information to enable model identification. Time series that lie on attractors that are not chaotic typically do not have enough dynamical information that would permit building a model without a priori knowledge. A number of examples (including real data) have been discussed and suggest that the new approach is useful in the estimation of NARMAX rational models from data.

\section{Acknowledgments}

The authors are grateful to CNPq for financial support. M. V. Corrêa's research is supported by ICMG.

\section{References}

Aguirre, L. A. [1997] "Recovering map static nonlinearities from chaotic data using dynamical models," Physica D100(1\&2), 41-57.

Aguirre, L. A. \& Jácome, C. R. F. [1998] "Cluster analysis of NARMAX models for signal-dependent systems," IEE Proc. Contr. Th. Appl. 145(4), 409-414.

Aguirre, L. A., Rodrigues, G. G. \& Mendes, E. M. [1997] "Nonlinear identification and cluster analysis of chaotic attractors from a real implementation of Chua's circuit," Int. J. Bifurcation and Chaos 7(6), 1411-1423.

Babloyantz, A. [1990] "Chaotic dynamics in brian activity," in Chaos in Brain Function, ed. Basar, E. (Springer-Verlag), pp. 42-48.

Babloyantz, A., Salazar, J. M. \& Nicolis, C. [1985] "Evidence of chaotic dynamics of brain activity during the sleep cycle," Phys. Lett. A111(3), 152-156.
Billings, S. A. \& Chen, S. [1989] "Extended model set, global data and threshold model identification of severaly nonlinear systems," Int. J. Contr. 50(5), 1897-1923.

Billings, S. A. \& Zhu, Q. M. [1991] "Rational model identification using an extended least-squares algorithm," Int. J. Contr. 54(3), 529-546.

Billings, S. A., Chen, S. \& Korenberg, M. J. [1989] "Identification of mimo nonlinear systems using a forward-regression orthogonal estimador," Int. J. Contr. 49(6), 2157-2189.

Brandstater, A. \& Swinney, H. L. [1987] "Strange attractors in weakly turbulent Couette-Taylor flow," Phys. Rev. A35(5), 2207-2221.

Briggs, K. [1987] "Simple experiments in chaotic dynamics," Am. J. Phys. 55, 1083-1089.

Chen, S. \& Billings, S. A. [1989] "Representations of nonlinear systems: The narmax model," Int. J. Contr. 49(3), 1013-1032.

Corrêa, M. V. [1997] Identification of Real Nonlinear Systems with NARMAX Rational Models, (in Portuguese) Master's Thesis, PPGEE, Universidade Federal de Minal Gerais, Belo Horizonte, Brasil.

Crutchfield, J. P., Farmer, J. D., Packard, N. H. \& Shaw, R. S. [1986] "Chaos," Sci. Am. December, 38-49.

Essex, C., Lookman, T. \& Nerenberg, M. A. H. [1987] "The climate attractor over short time scales," $\mathrm{Na}$ ture 326, 64-66.

Freeman, W. J. [1990] "Analysis of strange attractors in EEGs with kinesthetic experience and 4-d computer graphics," in Chaos in Brain Function ed. Basar, E. (Springer-Verlag), pp. 153-161.

Ghil, M., Kimoto, M. \& Neelin, J. D. [1991] "Nonlinear dynamics and predictability in the atmospheric sciences," Rev. Geophys. 29, 46-55.

Gilmore, R. [1998] "Topological analysis of chaotic dynamical systems," Rev Mod. Phys. 70(4), 1455-1529.

Gouesbet, G. \& Letellier, C. [1994] "Global vectorfield reconstruction by using a multivariate polynomial $l_{2}$ approximation on nets," Phys. Rev. E49(6), 4955-4972.

Govindan, R. B., Narayanan, K. \& Gopinathan, M. S. [1998] "On the evidence of deterministic chaos in ECG: Surrogate and predictability analysis," Chaos 8(2), 495-502.

Hegger, R., Kantz, H., Schmuser, F., Diestelhorst, M., Kapsch, R.-P. \& Beige, H. [1998] "Dynamical properties of a ferroelectric capacitor observed through nonlinear time series analysis," Chaos 8(3), 727-736.

Kennedy, M. P. [1992] "Robust op amp realization of Chua's circuit," Frequenz 46(3\&4), 66-80.

Korenberg, M. J., Billings, S. A., Liu, Y. \& McIlroy, P. J. [1988] "Orthogonal parameter estimation algorithm for nonlinear stochastic systems," Int. J. Contr. 48(1), 193-210. 
Krinsky, V. \& Pumir, A. [1998] "Models of defibrillation of cardiac tissue," Chaos 8(1), 188-203.

Leontaritis, I. J. \& Billings, S. A. [1985] "Input-output parametric models for nonlinear systems part ii: stochastic nonlinear systems," Int. J. Contr. 41(2), 329-344.

Letellier, C., Dutertre, P. \& Maheu, B. [1995] "Unstable periodic orbits and templates of the Rössler system: Toward a systematic topological characterizatio," Chaos 5(1), 271-282.

Lorenz, E. N. [1963] "Deterministic nonperiodic flow," $J$. Atmos. Sci. 20, 131-141.

Ménard, O., Letellier, C., Fang, H.-P., Maquet, J. \& Gouesbet, G. [1999] "Map reconstructions by using rational functions," in preparation.

Mendes, E. M. A. M. [1995] Identification of Nonlinear Discrete Systems with Intelligent Structure Detection. PhD Thesis, University of Sheffield, Sheffield, UK.

Mendes, E. M. A. M. \& Billings, S. A. [1998] "On overparametrization of global nonlinear discrete models," Int. J. Bifurcation and Chaos 8(3), 535-556.

Nicolis, C. \& Nicolis, G. [1987] "Evidence for climatic attractors," Nature 326(2), p. 523.

Rodrigues, G. G. [1996] Identification of Real Nonlinear Systems with NARMAX Polynomial Models, (in Portuguese), Master's Thesis, PPGEE, Universidade Federal de Minas Gerais, Belo Horizonte, Brazil.
Roschke, J. \& Basar, E. [1990a] "The EEG is not a simple noise: Strange attractors in intracranial structures," Chaos in Brain Function, ed. Basar, E. (SpringerVerlag), pp. 49-62.

Roschke, J. \& Basar, E. [1990b] "Correlation dimensions in various parts of cat and human brain in different states," Chaos in Brain Function, ed. Basar, E. (Springer-Verlag), pp. 92-109.

Rössler, O. E. [1976] "An equation for continuous chaos," Phys. Lett. A57(5), 397-398.

Skinner, J. E., Martin, J. L., Landisman, C. E., Mommer, M. M., Fulton, K., Mitra, M., Burton, W. D. \& Saltzberg, B. [1990] "Chaotic dynamics in brian activity," Chaos in Brain Function, ed. Basar, E. (Springer-Verlag), pp. 119-135.

Tse, C. K. [1994] "Flip biffurcation and chaos in threestate boost switching regulators," IEEE Trans. Circuits Syst. I 41(1), 16-23.

Zhu, Q. M. \& Billing, S. A. [1991] "Recursive parameter estimation for nonlinear rational models," J. Syst. Eng. 1, 63-76.

Zhu, Q. M. \& Billings, S. A. [1996] "Fast ortogonal identification of nonlinear stochastic models and radial basis functions," Int. J. Contr. 64(5), 871-886. 